\title{
BMJ Open Qualitative, multimethod study of behavioural and attitudinal responses to cochlear implantation from the patient and healthcare professional perspective in Australia and the UK: study protocol
}

To cite: Rapport F, Bierbaum M, McMahon C, et al. Qualitative, multimethod study of behavioural and attitudinal responses to cochlear implantation from the patient and healthcare professional perspective in Australia and the UK: study protocol. BMJ Open 2018;8:e019623. doi:10.1136/ bmjopen-2017-019623

- Prepublication history and additional material for this paper are available online. To view these files, please visit the journal online (http://dx.doi org/10.1136/bmjopen-2017019623).

Received 14 September 2017 Revised 5 March 2018 Accepted 26 April 2018

Check for updates

${ }^{1}$ Australian Institute of Health Innovation, Macquarie University, Sydney, New South Wales, Australia

${ }^{2}$ Macquarie University Centre for Implementation of Hearing Research, Sydney, New South Wales, Australia

${ }^{3}$ The HEARing Cooperative

Research Centre, Sydney, New South Wales, Australia

${ }^{4}$ South Wales Cochlear Implant Programme, Abertawe Bro

Morgannwg University Health

Board, Bridgend, UK

${ }^{5}$ Swansea University Medical

School, Swansea, UK

Correspondence to

Mia Bierbaum;

mia.bierbaum@mq.edu.au

\section{ABSTRACT}

Introduction The growing prevalence of adults with 'severe or greater' hearing loss globally is of great concern, with hearing loss leading to diminished communication, and impacting on an individual's quality of life (QoL). Cochlear implants $(\mathrm{Cl})$ are a recommended device for people with severe or greater, sensorineural hearing loss, who obtain limited benefits from conventional hearing aids $(\mathrm{HA})$, and through improved speech perception, Cls can improve the QoL of recipients. Despite this, utilisation of Cls is low.

Methods and analysis This qualitative, multiphase and multimethod dual-site study (Australia and the UK) explores patients' and healthcare professionals' behaviours and attitudes to cochlear implantation. Participants include general practitioners, audiologists and older adults with severe or greater hearing loss, who are HA users, $\mathrm{Cl}$ users and $\mathrm{Cl}$ candidates. Using purposive time frame sampling, participants will be recruited to take part in focus groups or individual interviews, and will each complete a demographic questionnaire and a qualitative proforma. The study aims to conduct 147 data capture events across a sample of 49 participants, or until data saturation occurs. Schema and thematic analysis with extensive group work will be used to analyse data alongside reporting of demographic and participant characteristics.

Ethics and dissemination Ethics approval for this study was granted by Macquarie University (HREC: 5201700539), and the study will abide by Australian National Health and Medical Research Council ethical guidelines. Study findings will be published through peer-reviewed journal articles, and disseminated through public and academic conference presentations, participant information sheets and a funders' final report.

\section{INTRODUCTION}

Prevalence of hearing loss

In 2006, hearing loss was ranked as the third highest cause of years lived with disability by the WHO. ${ }^{1}$ Hearing loss is strongly age related, and can lead people to experience diminished

\section{Strengths and limitations of this study}

The qualitative data, as well as survey responses, will be triangulated to corroborate and validate the findings, and ensure accurate and consistent reporting.

- Schema and thematic analysis will enhance the credibility of outputs through consensus building during group work activities.

- The qualitative study is built on a small-scale sampling technique, which, while normal practice in qualitative health research, suggests limited generalisability.

- This is an initial proof-of-concept study; however, findings will be applied to a wider population-based research study conducted in both Australian and UK contexts for global appeal.

communication, frustration and a sense of social isolation. ${ }^{2}$ Degrees of hearing loss are typically defined by an average of pure tone hearing thresholds (measured in decibels, $\mathrm{dB}$, as the minimum level of sound needed to be perceived) across different frequencies in the better ear. ${ }^{3}$

The global prevalence of severe or greater hearing loss $(\geq 65 \mathrm{~dB})$ among people aged 15 years or older is $1.2 \%$ for men and $1.0 \%$ for women. ${ }^{4}$ It is estimated that $74 \%$ of the world's population of 7550 million people is aged over 15 years (equating to approximately 5.6 billion people), ${ }^{5}$ which suggests that over 60 million people globally have severe or greater than severe (known from hereon in as 'severe or greater') hearing loss. These prevalence estimates, however, are based on studies conducted between 1973 and 2010, and given the shift towards an ageing population, ${ }^{6}$ they may be an under-representation of current prevalence. ${ }^{7}$ In 2005, approximately one in 
five adults (3.5 million) in Australia had hearing loss, ${ }^{8}$ and $11 \%$ of these ( 400488 adults) were estimated to have severe or greater hearing loss in at least one ear, ${ }^{8}$ with the frequency in Australian adults projected to exceed 573000 by $2020 .^{8}$

\section{Hearing loss devices}

Hearing aids (HA) are commonly recommended for adults with hearing loss. These are electronic devices placed in the outer ear that selectively amplify different frequencies in a compressive non-linear manner, reproducing the activity of cochlear outer hair cells. HAs rely on a sufficient number of remaining healthy hair cells in the cochlea (ie, the inner ear) to transduce the amplified acoustic signal into an electric signal that travels to the auditory centres in the brain via the auditory nerve. More damaged or non-functioning hair cells in the cochlea leads to greater magnitudes of hearing loss, further limiting the effective transduction of an amplified signal to the auditory nerve. As such, amplification does little to support people with more severe sensorineural hearing loss. ${ }^{9}$ For this group, a cochlear implant (CI) can be recommended to bypass damaged hair cells in the cochlea with a surgically implanted electrode array, and significantly restore the perception of sound. With CIs, speech and environmental sounds are captured by an externally worn speech processor, converted into an electric signal and transmitted to the electrode array in the cochlea, transducing the signal to the cochlear nerve. ${ }^{10}$

\section{Benefits of Cls}

The literature on hearing loss highlights how a large proportion of adults with postlingual sensorineural severe or greater hearing loss (ie, hearing loss caused by damage to hair cells in the cochlea, which has occurred after the development of language) benefit from CIs, ${ }^{11-13}$ including improved speech perception and enhanced quality of life (QoL)..$^{1214-16}$

Unless fluent in sign language and engaged in a deaf community, adequate hearing is a vital element of social and emotional well-being, self-efficacy and connectedness, ${ }^{17}$ and important for occupational well-being. ${ }^{18}$ There is a clear association between hearing loss and dementia ${ }^{19}$ and depression, ${ }^{20}{ }^{21}$ reinforcing the importance of ensuring people are provided with timely access to appropriate resources. In addition to improving listening abilities and QoL, CIs are a cost-effective intervention, with a cost utility of nearly US $\$ 10000$ per quality-adjusted life year. ${ }^{22}$ This takes into account direct costs (eg, the cost of the device) and indirect costs (eg, the cost for loss of wages or productivity). ${ }^{22}{ }^{23}$ Despite these factors, utilisation of CIs globally remains low among the adult population. ${ }^{24}$

\section{Utilisation of Cls}

Prevalence of severe or greater hearing loss and CI utilisation rates are not well documented. It is important to note that when comparing data from different countries, some literature focuses on the adult population, some on the child population and some on a mixture of the two, while the age and the severity of hearing loss criteria listed for adults can also vary. In the USA, in 2009, it was estimated that 1.2 million adults and children with severe to profound hearing loss were eligible for a CI, but less than $6 \%$ had obtained one, while it was estimated that $90 \%$ of this population used HAs. ${ }^{25}$ In the UK in 2009, approximately 613000 adults were estimated to have severe to profound hearing loss, including $3 \%$ and $8 \%$ of adults over 50 and 70 years, respectively, many of whom may benefit from a CI. ${ }^{26}$ Although the annual number of CI surgeries in the UK's adult population is gradually increasing, between April 2016 and March 2017, there were 919 CIs implanted in adults unilaterally, bilaterally simultaneously and bilaterally sequentially. ${ }^{27}$ This indicates low utilisation rates in adults, particularly when compared with the child population, where, in 2011, $94 \%$ of eligible children (aged 16 years or under) used CIs ${ }^{28}$ as a result of the universal newborn hearing screening programme. ${ }^{28}$

In Australia in 2006, 87634 people aged 15 years and over were estimated to have at least severe hearing loss in their better ear. ${ }^{8}$ In 2014, Cochlear, one of several CI manufacturers in Australia, estimated the number of CI devices implanted in Australia in children and adults to be $10370^{29}$; however, this figure is likely an under-representation of the total number of CI devices implanted as it may not include CI devices produced by other manufacturers. In 2014-2015 (financial year), 1498 CI procedures took place in Australia in both children and adults, ${ }^{30}$ an increase in the annual number of CI surgeries since 2011-2012 of $1177 .{ }^{31}$ While it has been estimated that less than $10 \%$ of those eligible for a CI have received the device, ${ }^{8}$ the exact CI utilisation rate is unknown. In particular, CI candidacy guidelines in Australia currently rely on speech recognition ability while using HAs, and not on the severity of hearing loss as defined by hearing thresholds. ${ }^{9}$ No prevalence data are available where the hearing loss was defined in terms of hearing (dis)ability instead of hearing thresholds. With these discrepancies of reporting, further research could help determine current utilisation rates in the adult Australian population.

\section{Healthcare professional perspectives}

While research regarding the reasons for the low rates of CI utilisation ( $10 \%$ or less) globally is limited, ${ }^{24}$ suggestions include: a lack of awareness about the benefits of CIs in the general population; healthcare professionals' limited knowledge of CI candidacy criteria; and low referral rates to specialist hearing services. ${ }^{32}{ }^{33}$ Audiologists have been found to be positive in their attitudes towards the benefits of CIs for adults with postlingual deafness, yet some healthcare professionals are hesitant about the benefits for older adults. ${ }^{29} 34$ This may stem from assumptions that rehabilitation will be hindered by age-related cognitive decline and the deterioration of the auditory pathway, as well as concerns about the risks 
associated with general anaesthesia in the older adult population. ${ }^{34}$ Concerns persist, despite the fact that US studies have shown that CIs are effective in the older adult population (60 years or older) ${ }^{35}$ and that benefits for this age group are not significantly different from benefits in younger age groups. 3536

Healthcare professionals' levels of training, knowledge, experience and their relationship with CI programmes, as well as uncertainty about which patients are appropriate CI candidates, and when and how to make a referral to a CI service centre, are also said to determine referral behaviour and contribute to low CI utilisation. ${ }^{29}{ }^{32}$ Education programmes for healthcare professionals about the benefits of CIs, with the provision of information about candidacy criteria, have shown potential in increasing referral rates, ${ }^{24}{ }^{33}$ but have yet to be fully established at national levels.

\section{Reported barriers to utilisation of hearing devices and rehabilitation in adults over the age of $\mathbf{5 0}$ years}

Studies from the UK, Australia and the USA have reported that some adults over the age of 50 with hearing loss delay seeking assistance from a healthcare professional ${ }^{37}$ because they are: (A) in denial about the severity of their hearing loss, ${ }^{38}$ (B) concerned about the perceived inconvenience of accessing hearing rehabilitation $^{39}$ and (C) worried about the cost of a hearing device and its ongoing maintenance. ${ }^{39}$ There are also indications that older adults with hearing loss often associate hearing technologies, such as HAs and CIs, with the stigma of ageism, or disability, ${ }^{39-41}$ while competing comorbidities are identified as a barrier to accessing hearing rehabilitation, as other healthcare conditions take priority. ${ }^{32} 3842$

Despite these reported barriers to healthcare utilisation, more targeted research is needed to clarify how CIs are perceived, both in the adult population utilising CIs and in the adult population with severe or greater hearing loss who are not yet utilising CIs. In addition, research is required to determine how access to services is discussed between patients and healthcare providers, including general practitioners (GP) (who refer patients to hearing support services) and HA audiologists (referred to here as 'audiologists'), and how discussions affect the decisions made and patient pathways taken through healthcare systems. It is important to note that the current CI candidacy criteria differ across countries. Furthermore, there is scant literature on healthcare providers': (A) perceptions of the advantages and disadvantages of CIs, (B) knowledge of CI candidacy and (C) views about facilitators and barriers to CIs, to inform research for service improvement ${ }^{9}$ and create more equitable care provision. This current gap in the literature warrants further attention, to better understand the barriers and facilitators, in order to enable a greater proportion of individuals who would benefit from CIs to gain access to these devices, in order to enhance their QoL.

\section{METHODS AND ANALYSIS}

\section{Study design}

We propose a qualitative, multiphase, multimethod (in this case more than one qualitative method) and dualsite study, undertaken concurrently in Australia and the UK to explore patients' and healthcare professionals' behaviours and attitudes to cochlear implantation. The study design is based on the Consolidated Criteria for Reporting Qualitative Research. ${ }^{43}$ This 1-year study will run from June 2017 to June 2018.

\section{Study context}

In Australia, HAs and unilateral CIs are available with public funding through the Australian Government Hearing Services Program, State Government funding and the Department of Veterans' Affairs; for people who meet the eligibility criteria. The number of CIs that are publicly funded each year is limited, and varies state by state, resulting in waiting lists. ${ }^{44}$ Private health insurance funds can be used to reimburse implantation of the second ear. ${ }^{2345}$

In the UK, HAs are available through the publicly funded National Health Service (NHS) or are available from private dispensers via self-funding or private health insurance. Unilateral CIs are available through the NHS for adults who meet the eligibility criteria specified by the National Institute for Health and Care Excellence. ${ }^{26}$ Alternatively, CIs may be self-funded or available through private health insurance plans. ${ }^{46}$

\section{Study objectives}

In order to explore the behaviours and attitudes to cochlear implantation from the healthcare professional and patient perspectives, the study objectives are to: (A) determine perceptions of barriers and facilitators associated with cochlear implantation in adults aged 50 years and older, with a postlingual and severe or greater sensorineural hearing loss; (B) assess patients' and healthcare professionals' behaviours and attitudes to cochlear implantation; and (C) clarify how attitudes and behaviours impact on patient pathways through healthcare services.

\section{Research team}

The research team is made up of researchers with varying levels of experience conducting qualitative research within the audiology field. The lead data collection researcher MB (Research Officer, MPH) has experience conducting qualitative and quantitative health research and has been trained in focus group facilitation and interviews. She does not work within the field of hearing healthcare, removing potential researcher bias during data collection. The co-researchers include FR (Professor, PhD), who is a significantly experienced qualitative researcher, JB (Professor, PhD), an extensively experienced implementation science researcher, CM (Professor, PhD) and IB (Research Fellow, PhD), who are considerably experienced audiologists and audiology researchers, AL (Senior 
Research Fellow, PhD), an experienced consumer informatics researcher, and SH (Speech and Language Therapist, $\mathrm{PhD}$ candidate), who has experience working and conducting qualitative research within the audiology field, and is trained in focus group facilitation. The participants will be provided with an information sheet detailing the purpose and aim of the study, as well as the contact details of the data collection researcher (MB) and study lead (FR).

\section{Participants}

The Australian participant cohort will include: (1) GPs; (2) audiologists; and (3) adults over the age of 50 with bilateral (both ears) severe or greater postlingual hearing loss selected according to two classifications: (A) bilateral or unilateral CI users and (B) HA users or CI candidates (in the process of being assessed for a CI). The UK cohort will include audiologists only to provide a healthcare professional comparison group from an international perspective. The UK site will concentrate on audiologists' practices, behaviours and attitudes to ascertain the CI referral process, related patient pathways and care provision for the older adult population.

\section{Participant inclusion criteria}

Patient participants will be: 50 years or over; have bilateral severe or greater postlingual sensorineural hearing loss; be proficient in English; and be willing and able to engage in focus group discussions and complete a written demographic questionnaire and qualitative proforma (open-ended survey).

GPs and audiologists must be currently working in their field and have had experience consulting with the target populations. The study will aim to recruit healthcare professionals with a variety of experiences working within their field and with people with a hearing loss.

\section{Recruitment}

The study aims to conduct 147 data capture events across a sample of 49 participants, or until data saturation occurs ${ }^{47}$ (table 1). In order to achieve these numbers, recruitment of GPs, audiologists and adults with severe or greater hearing loss will continue until February 2018 or until saturation is achieved. Promotional flyers for audiologists will be distributed to audiology clinics and hearing health conferences. Promotional flyers will be sent to GP clinics via professional network e-newsletters. Promotional flyers for adults with hearing loss will be distributed to hearing associations for display on their websites, placement in newsletters and placement on social media sites. Audiologists will also provide eligible clients with the promotional flyers. Flyers will include general study information and research team contact information. Using this approach minimises direct researcher contact with potential participants and reduces the possibility of researcher coercion in the recruitment process. Researchers will have no prior relationships with participants. Recruitment of audiologists, GPs and adults with hearing loss will be Australia-wide. Recruitment of audiologists in the UK will be UK-wide.

In this study, purposive time frame sampling ${ }^{48-50}$ will be employed to ensure a wide mix of participants. In the case of patients, purposive sampling means a mix of gender, socioeconomic class, age and ethnicity. In the case of professionals this means a mix of gender, service location (rural and metropolitan areas) and patient group served. Eligible study participants will be enrolled into each focus group in the order in which they respond to the promotional flyers and contact the research team. Time frame sampling encourages researchers to outline a predefined recruitment period thus ensuring eligible individuals have an equal opportunity of being enrolled during that time frame. This removes the possibility of recruitment becoming opportunistic, ${ }^{51}$ and while it is acknowledged that self-selection may introduce a degree of bias, it also ensures participants are willing to engage in the study and provide detailed information about their experiences.

Once individual eligibility is established, and informed consent given, participants will be allocated to a study

Table 1 Frequency of data capture events for the pilot and principal study per participant group

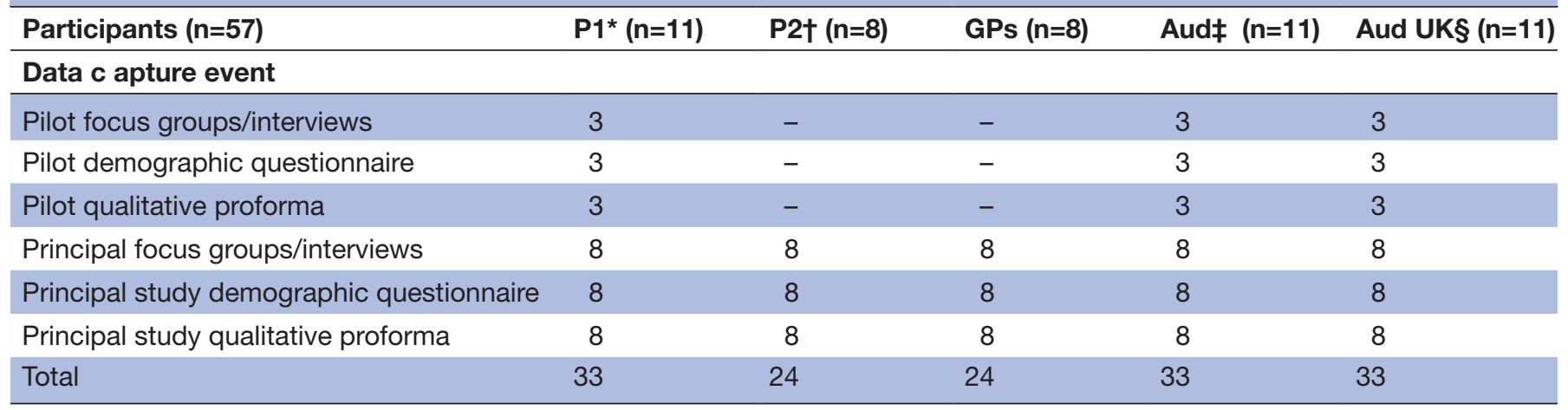

${ }^{*} \mathrm{P} 1$ : Cochlear implant user.

†P2: HA user and cochlear implant candidate.

$\ddagger$ Aud: HA audiologist in Australia.

§Aud UK: HA audiologist in the UK.

GP, general practitioner; HA, hearing aid. 
focus group, until each of the six planned focus groups have reached maximum capacity (10 participants, $20 \%$ above the required participant number $(n=8))$. Overestimating participant recruitment will ensure that sample saturation can be achieved, with a well-recognised attrition rate of $20 \%$ for focus group attendance. ${ }^{52}$ The sample size has been determined according to the literature on optimal focus group numbers for full participation. The sample size will also encourage participants to voice their opinions freely. ${ }^{53-55}$

Three audiologists and three CI users will be invited to participate in two separate pilot focus groups in Australia, and three audiologists will be invited to participate in a focus group in the UK. The questions, questionnaires and proformas will be similar across the patient cohorts and similar across the healthcare professional cohorts. The pilot focus groups and pilot interviews will test the acceptability, timeliness and comprehensiveness of the questions, and ensure the methodology complements the study's aims and objectives. The same recruitment, data collection and analysis methods used for the principal study will be used for the pilot study.

Forty principal study participants and nine pilot study participants will be enrolled across Australian and UK sites to enable a comprehensive exploration of the subject matter. This sample is entirely appropriate for a qualitative study ${ }^{54}$ aimed at eliciting in-depth, rich and 'thick' descriptions of 'lived experience', ${ }^{56}$ and lends itself to work with different population groups.

\section{Data collection}

The 1-year study is supported by two stages of data collection (figure 1).
Stage 1

Stage 1: Literature review, pilot and principal study focus groups and interviews, as well as the completion of demographic questionnaires.

\section{Literature review}

Stage 1 commences with a literature review of the current research around barriers and facilitators to CI use, associated health service provision and patient pathways through healthcare systems. The results will inform the development of the focus group questions and demographic questionnaire.

\section{Focus groups}

Two pilot focus groups will be conducted in Australia with audiologists and CI users, one pilot focus group will be conducted in the UK with audiologists, and five principal study focus groups (four in Australia with CI users, HA users and CI candidates, GPs, audiologists; and one in the UK with audiologists) will be conducted during stage 1 .

The focus groups with adults with hearing loss will cover questions regarding:

- Hearing and QoL.

- Information and support received.

- Hearing devices used.

- Motivators and facilitators to using CIs.

Focus groups with healthcare professionals will include questions regarding:

- Knowledge about CIs and candidacy criteria.

- Experience of discussing hearing loss and CIs with patients.

- Perception of CIs and how they impact patients.

- Types of support needed to discuss hearing loss with patients.

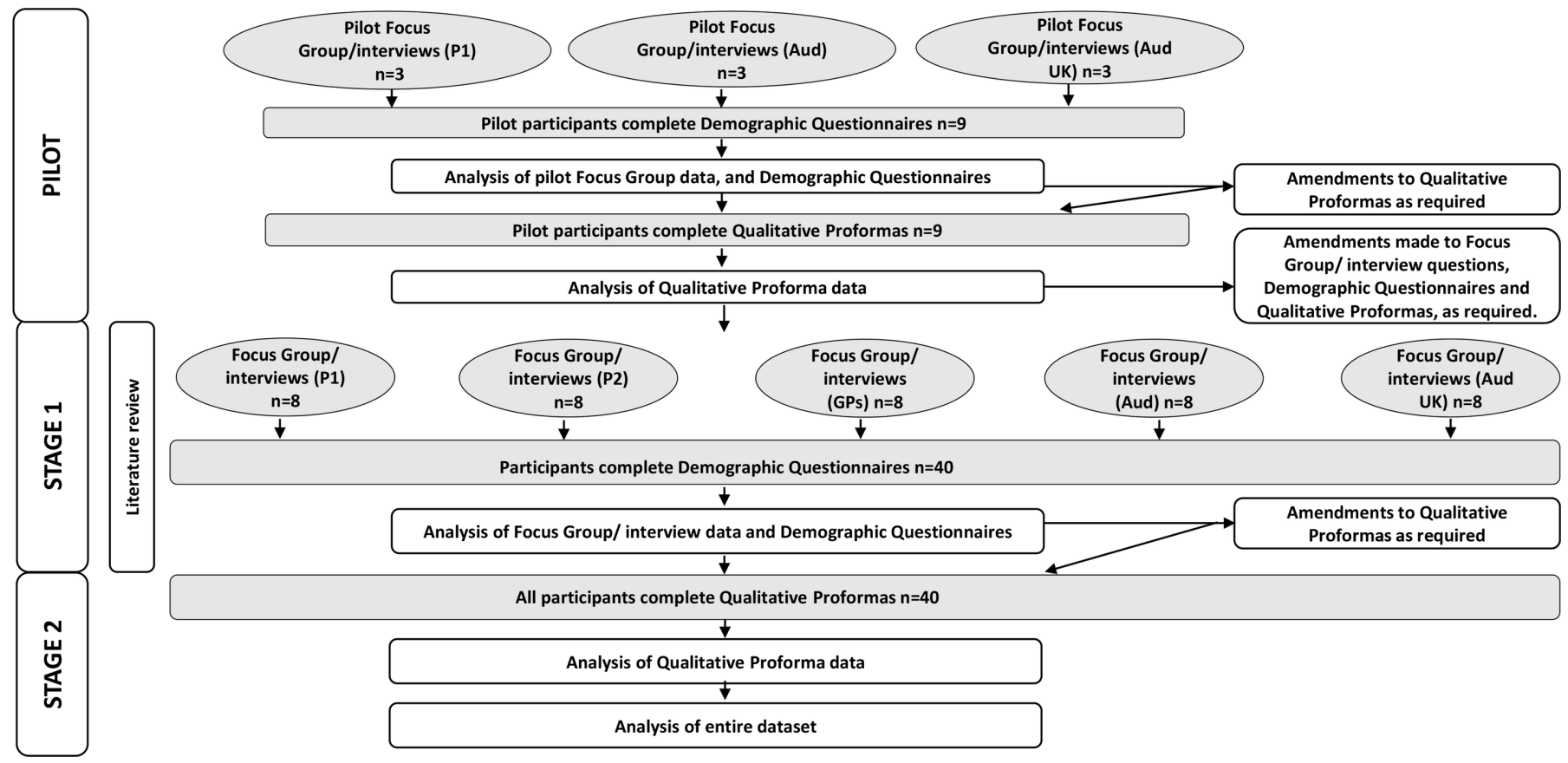

Figure 1 Study plan. GP, general practitioner. 
- Shared care with hearing services and hearing specialists.

The study researcher (MB) will facilitate the Australian focus groups in a meeting space within the university, with an observer present (noting group dynamics, body language and gestures, and managing audio recording equipment). ${ }^{53}$ The UK chief investigator ( $\mathrm{SH}$ ) will conduct the UK audiologist focus group/interviews in meeting spaces at participants' workplaces, community halls, or in meeting rooms at the local health board, as well as via Skype if required, and will follow the same methods to those used in Australia to ensure consistency.

Each focus group will be approximately an hour in length and will be audio-recorded. During focus groups, participants will be arranged around a table, so they can see each other's faces. Participants will be provided with a copy of the 'Rules of Engagement' before focus groups commence, which advise that participants should respect each other's opinions, be willing to listen to others and to speak in turn. ${ }^{53}$ Participants will also be sent the focus group questions ahead of time, to assist with understanding during the live group events. In addition, communication support in the form of either remote, live, real-time captioning by a stenographer, ${ }^{58}$ a wireless assistive hearing device system, or an Auslan (Australian Sign Language) interpreter ${ }^{59}$ will be available if required. Provision of such support is considered best practice when running focus groups with people who have hearing loss to enable conversations to be accessible and comprehensible for all participants. ${ }^{58}$ Participants will be reminded that focus groups will be recorded unless objections are voiced, in which case written notes alone will be taken. Transcriptions will include pseudonyms to minimise the possibility of any participant being identified, ${ }^{57}$ and participants will be advised of preparations for data confidentiality and personal anonymity.

All participants will be offered a stipend as a gesture of appreciation for their time and refreshments will be provided.

\section{Interviews}

Eligible participants unable to attend a focus group but keen to participate will be interviewed by phone, via Skype or email, depending on their preference. The videoconferencing option on Skype enables participants to see the speaker, to lip-read and see facial gestures. Focus group questions will be sent out in advance and used as interview questions. Participants will also receive a consent form and demographic questionnaire to be completed before the phone interview, Skype interview or email communication takes place. All interviews will be audio-recorded and transcribed.

\section{Demographic questionnaires}

Following focus groups and interviews (irrespective of data capture method), participants will be asked to complete a demographic questionnaire designed to provide contextual information about individual service provision or receipt (see online supplementary file). Two questionnaires will be produced; one for healthcare professionals and one for patients, with multiple-choice, open-ended questions.

The Patient Demographic Questionnaire will include questions about the participant's gender, age, health insurance status, home postcode, comorbidities, hearing loss, hearing device use and information acquisition experiences.

The Healthcare Professional Demographic Questionnaire will include questions about the professional's gender, age, occupation, workplace, work postcode, years of practice, public or private healthcare service provider status, frequency of working with patients with hearing loss and information provision experience.

\section{Analysis of stage 1 data}

Focus group, interview and demographic questionnaire data will be analysed to inform the development of stage 2. All audio recordings of focus groups and interviews will be transcribed as they are completed, along with field notes, to ensure the team's early immersion in data. Analysis will be conducted continuously, and results built on iteratively, while data are being collected. This way, any changes to the data collection tools in phase 2 that may be necessary can be undertaken early on during the study. By starting analysis as data are collected we will comply with an inductive approach to data analysis, ${ }^{60}$ be better informed and able to facilitate clearer dialogue during the focus groups and the interviews.

Qualitative schema analysis and thematic analysis techniques will be used, ${ }^{61}$ which are common methods for the analysis of focus group and interview transcripts, where extensive data contain multiple voices (figure 2). Schema analysis allows team members to create individual and group overviews of texts, while at the same time revealing essential textual elements, derived in a summarised form, to highlight key notions or concepts embedded in texts. Schema analysis lends itself to a group working approach and helps validate findings through consensus building activities, where critical findings are discussed and agreed upon by the group. ${ }^{61-63}$ Group work analysis will include the study team: authors MB, FR, CM, IB, SH and AL. UK-based researcher, SH, will participate in all Australian-based team work discussions via Skype or email. In addition, the UK researcher will be in regular contact with the Australian project officer (MB) to ensure data collection is conducted in the same way across the two sites. Demographic questionnaire data will be analysed using descriptive statistical techniques. ${ }^{64}$ Demographic data will be described to provide context about the participants.

The UK data will be analysed using the same methods as the Australian data. Any similarities and differences between the two cohorts will be highlighted, and comparisons about service provision will be made between the audiologist data from the two sites. The UK sample will offer rich comparative healthcare provider detail, and insights into differences between services in both sites. 
The authors will compare audiologists' perceptions, and experiences across the sites, with plans to include other stakeholder groups in a follow-on study, to provide more comparative detail.

Stage 1 data will elicit information about, but not restricted to: support for cochlear implantation; the process of CI candidacy; consultation and negotiation; types of information provision; professional practices; misconceptions and misunderstandings; effects of HAs and CIs on QoL; daily routines and relationships; concerns, fears and anxieties; patient pathways through healthcare systems; patient and professional information needs; and long-term aspirations for hearing health.

\section{Stage 2}

Stage 2: Development of the qualitative proformas will be informed by stage 1 analysis and the results of the literature review. Stage 2 is designed to build understanding, investigate data inconsistency and expand findings, while corroborating earlier data sets.

\section{Qualitative proformas (open-ended surveys)}

Each participant will be sent a qualitative proforma to complete (see online supplementary file). Qualitative proformas are brief, open-ended surveys containing limited choice questions (approximately four to six), for participants to qualify their responses from other data capture events. In this study, qualitative proformas will be designed through team discussion, and personalised for each principal focus group cohort.
Qualitative proformas will include questions about:

- Information provision/receipt about CIs.

- Shared decision-making about hearing health.

\section{Analysis of qualitative proformas}

Qualitative proformas will be analysed using thematic analysis techniques ${ }^{65}$ to disclose key themes and their concomitant categories. Individual and group qualitative proforma analysis will lead to the development of a thematic analytic framework. Comparisons will be drawn between the UK and Australian audiologists' proformas to highlight differences in referral pathways and knowledge about CIs between the two sites. Thematic analysis teamwork will require all team members to contribute to, and agree on the final thematic frameworks, sharing decisions about key issues arising.

Open coding will be conducted initially during data collection, to break down the data into discrete ideas, followed by axial coding which will help classify the discrete codes, and categories into broader themes, once data collection is complete. ${ }^{66}$ NVivo Pro 11 software V.11, 2015, will be used to code the data, for the thematic analysis, to derive themes and categories, to enhance the rigour of the working methods and trustworthiness of results, through systematic and transparent coding of data. ${ }^{67}$

Data from all study stages will be treated corroboratively, with each element informing the next, and leading to a comprehensive data triangulation (testing one source of data against other sources of data to

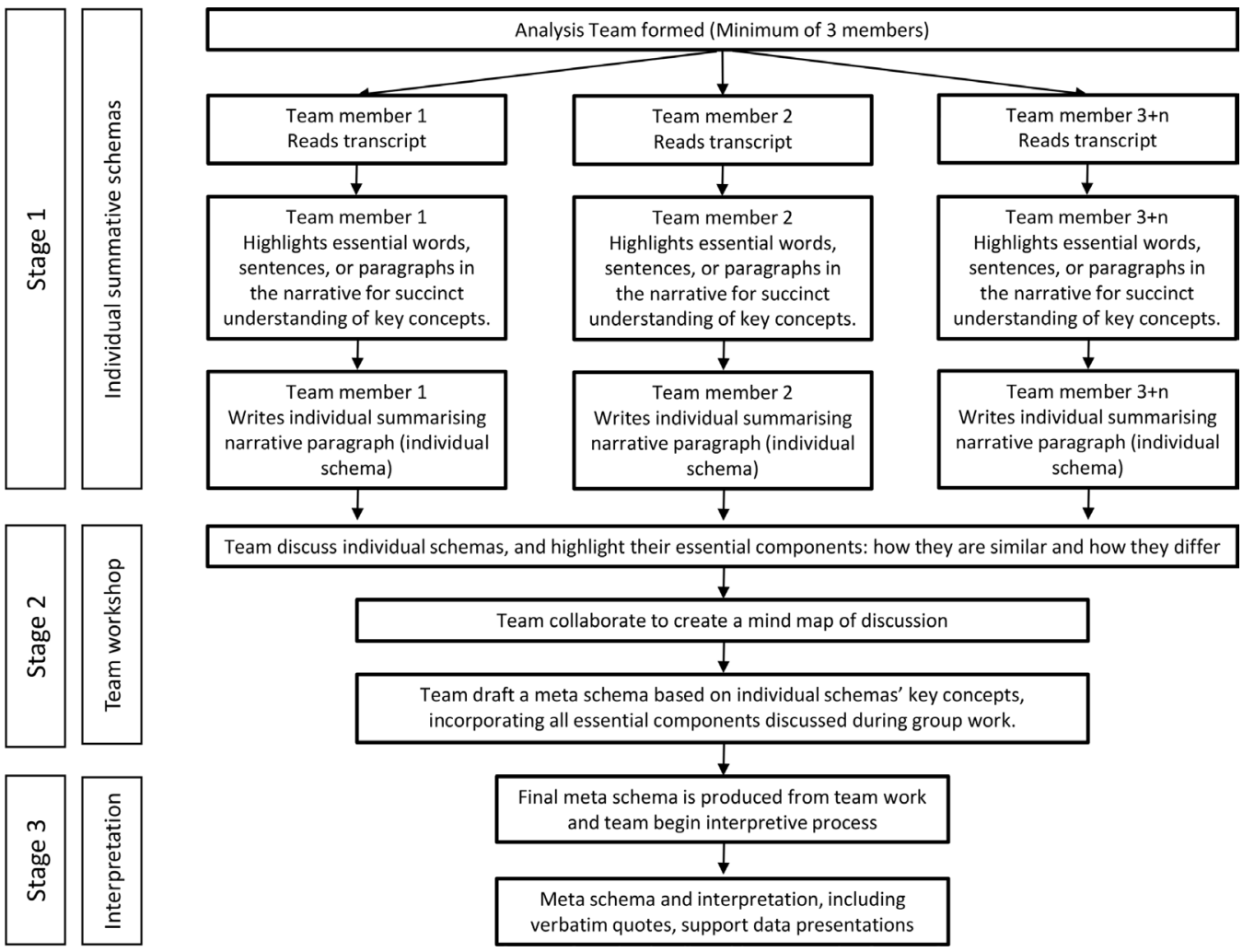

Figure 2 Simplified presentation of schema analysis, an in-depth teamwork analysis process (adapted from Rapport et al [63]). 
cross-validate or explain discrepancies in sources), ${ }^{68-71}$ verifying results and strengthening the study's validity. The multimethod data collection and analysis techniques are designed to encourage nuanced and detailed understandings of the experiences of CI users, HA users and CI candidates, GPs and audiologists. The findings will be generated from the transcripts and proforma data using an inductive approach to data analysis. This refers to the way that theory 'emerges' from the data, which is dealt with 'from the ground up', with findings grounded in the raw material and meaning revealed iteratively. An inductive approach to data analysis in this study will enable researchers to develop a thematic framework based on the key themes and categories arising within the data. ${ }^{60}$

\section{Presentation of results}

Results from the focus groups, interviews and proformas will be presented as themes and their concomitant categories, with verbatim quotations embedded in the narrative that describes the themes, to support and add authenticity to the research group's interpretations.

\section{Enhancing the trustworthiness of the research}

The rigour of data will be achieved by applying Lincoln and Guba's theory of trustworthiness to attain credibility, transferability, dependability and confirmability. ${ }^{72}$ The pilot phase of the study, where focus group questions, questionnaires and proformas are tested on a small sample of participants, adds to the trustworthiness of the data, as it will validate whether the tools accurately measure and collect the information as intended. ${ }^{70}$

The use of data triangulation, multiple methods of data collection, across multiple cohorts with differing perspectives, as well as using multiple researchers to code the data will ensure credibility of findings, and make sure that the findings represent the attitudes of the participants, and are not biased by the researcher. The sample of participants will be diverse and encompass healthcare professionals from a range of settings and experiences, as well as patients from a range of age groups, experiences and locations. This will provide data representing the diverse views of the participants, leading to findings that will be generalisable. Recruitment, data collection, data analysis and results will be described in 'thick' detail, providing opportunity for the research to be replicated by other researchers, ensuring dependability.

Multiple researchers from the team will code the data, and validate the analytic framework to ensure the interpretation of data is not biased by a single researcher's perspective, but grounded by the contents of the data. ${ }^{73}$ These group working methods will ensure that the narrative summaries derived from the thematic frameworks are valid and trustworthy according to the whole team's views.

\section{Patient and public involvement}

There is a gap in the literature regarding the patient and healthcare professional perspective on barriers and facilitators to cochlear implantation. While there is abundant evidence regarding the experience of adults with hearing loss and HAs, there is little that specifically addresses the complex decision-making around CIs. The research question and outcome measures were developed through consultation with research team members who are audiologists and speech therapists, who ensured the study question was relevant given their experience in hearing health, and to ensure the outcome measures were appropriate. They also acted as representatives of the healthcare professional cohort, providing feedback about their experiences as professionals, and guiding the development of the study. Pilot focus groups and interviews were also conducted with patients and healthcare professionals to ensure the patient perspective was embedded from the outset and that assessment tools were acceptable and gathered appropriate data. All results will be disseminated to study participants upon completion of the project through information sheets and an executive summary. These documents will also be distributed to hearing associations, and participating GP and audiology clinics.

\section{ETHICS AND DISSEMINATION}

Ethical approval for the study has been granted by the Macquarie University Human Research Ethics Committee (HREC) (approval number 5201700539). All data collection will be conducted in accordance with the National Health and Medical Research Council ethical guidelines, ${ }^{74}$ and will adhere to the principles of the Macquarie University Human Research Ethics Committee. All participants will provide informed consent before participating. Participation will be voluntary, and participants will be able to withdraw at any time with no risks anticipated. If undue distress is caused by any aspect of this study, an appropriate healthcare professional or researcher will respond to the needs of the participant, with counselling services available if necessary.

\section{Data storage and protection}

All study materials will be deidentified and data will be stored on password-protected computers, in locked filing cabinets and locked offices on university premises, separate from participant identifiers. All data will be destroyed 7 years after completion of the study, in accordance with standard ethical guidelines. All anonymised UK and Australian data will be shared via secure password-protected online university storage, and security and anonymity of data will be upheld.

\section{Dissemination}

Findings will be reported to the funders and disseminated widely through international, peer-reviewed, open-access journal publications, public and academic presentations, oral and poster presentations at scientific conferences and a service user group information sheet. 


\section{SIGNIFICANCE AND IMPACT OF THE STUDY}

This will be the first Australian study to reveal, using a range of innovative methods, behavioural and attitudinal aspects of hearing service provision for older adult HA and CI users compared with a UK service example. The study will disclose a wide range of public and professional perspectives on hearing loss and views on barriers and facilitators to cochlear implantation in complex healthcare systems. It will be underpinned by a comprehensive overview of the international literature on the topic and indicate motivators and demotivators to service provision for prospective CI users.

Individual patients may find the process of self-reflection validating, while sharing insights with others in a similar situation may establish a supportive community. Healthcare professionals and patients will mutually benefit from clearer information on hearing health. At the end of the study, all participants will receive a summary information sheet, and links to useful sources of information. The process may assist healthcare professionals in understanding more about the patient experience, and how to move patients effectively through CI candidacy channels to better support their needs.

This study will lead to data that is representative of both healthcare professional and patient perspectives, illuminating current hearing health pathways in Australia and the UK, highlighting gaps in current services and raising awareness of CI users' needs. This will be the first stage in developing a longitudinal, pan-Australian study, with and international arm, leading to implementable outcomes to increase public awareness of hearing loss and enhance consumer support, while offering healthcare professionals clear referral guidelines.

Acknowledgements The authors would like to thank all participants who contributed to the study and shared their experiences with hearing loss.

Contributors FR led the conception of the study design, and the acquisition, drafting and revision of the work (for important intellectual content). MB contributed to the design, drafting and revision of the work. $\mathrm{CM}, \mathrm{IB}, \mathrm{AL}, \mathrm{JB}$ and $\mathrm{SH}$ contributed to the conception of the study design, and the acquisition and revision of the work (critically for important intellectual content). All authors provided final approval of the version published, and agree to be accountable for all aspects of the work in ensuring that questions related to the accuracy or integrity of any part of the work are appropriately investigated and resolved.

Funding This work is supported by Cochlear Macquarie University Partnership (MQ-Cochlear).

Competing interests None declared.

Patient consent Obtained.

Ethics approval Macquarie University Human Research Ethics Committee (approval number 5201700539)

Provenance and peer review Commissioned; externally peer reviewed.

Open Access This is an Open Access article distributed in accordance with the Creative Commons Attribution Non Commercial (CC BY-NC 4.0) license, which permits others to distribute, remix, adapt, build upon this work non-commercially, and license their derivative works on different terms, provided the original work is properly cited and the use is non-commercial. See: http://creativecommons.org/ licenses/by-nc/4.0/

(C) Article author(s) (or their employer(s) unless otherwise stated in the text of the article) 2018. All rights reserved. No commercial use is permitted unless otherwise expressly granted.
REFERENCES

1. Vos T, Barber RM, Bell B, et al. Global, regional, and national incidence, prevalence, and years lived with disability for 301 acute and chronic diseases and injuries in 188 countries, 1990-2013: a systematic analysis for the Global Burden of Disease Study 2013. Lancet 2015;386:743-800.

2. Sindhusake D, Mitchell P, Smith W, et al. Validation of self-reported hearing loss. The blue mountains hearing study. Int J Epidemiol 2001;30:1371-8.

3. Clark JG. Uses and abuses of hearing loss classification. ASHA 1981;23:493-500.

4. Stevens G, Flaxman S, Brunskill E, et al. Global and regional hearing impairment prevalence: an analysis of 42 studies in 29 countries. Eur $J$ Public Health 2013;23:146-52.

5. United Nations DoEaSA, Population Division. World population prospects: the 2017 revision, key findings and advance tables. Working Paper No. ESA/P/WP/248. New York, USA: United Nations DoEaSA, 2017.

6. United Nations DoEaSA, Population Division. World population ageing 2015 (ST/ESA/SER.A/390). New York, USA: United Nations DoEaSA, 2015.

7. Goman AM, Reed NS, Lin FR. Addressing estimated hearing loss in adults in 2060. JAMA Otolaryngol Head Neck Surg 2017;143:733-4.

8. Access Economics. The economic impact and cost of hearing loss in Australia. Canberra: Access Economics, 2006. A report by Access Economics P/L.

9. Leigh JR, Moran M, Hollow R, et al. Evidence-based guidelines for recommending cochlear implantation for postlingually deafened adults. Int J Audiol 2016;55(sup2):S3-S8.

10. Nikolopoulos TP, Vlastarakos PV. Treating options for deaf children. Early Hum Dev 2010;86:669-74.

11. Mohr PE, Feldman JJ, Dunbar JL, et al. The societal costs of severe to profound hearing loss in the United States. Int J Technol Assess Health Care 2000;16:1120-35.

12. Gaylor JM, Raman G, Chung M, et al. Cochlear implantation in adults: a systematic review and meta-analysis. JAMA Otolaryngol Head Neck Surg 2013;139:265-72.

13. Bittencourt AG, Ikari LS, Della Torre AA, et al. Post-lingual deafness: benefits of cochlear implants vs. conventional hearing aids. Braz J Otorhinolaryngol 2012;78:124-7.

14. Contrera KJ, Betz J, Li L, et al. Quality of life after intervention with a cochlear implant or hearing aid. Laryngoscope 2016;126:2110-5.

15. Mäki-Torkko EM, Vestergren $\mathrm{S}$, Harder $\mathrm{H}$, et al. From isolation and dependence to autonomy-expectations before and experiences after cochlear implantation in adult cochlear implant users and their significant others. Disabil Rehabil 2015;37:541-7.

16. Damen GW, Beynon AJ, Krabbe PF, et al. Cochlear implantation and quality of life in postlingually deaf adults: long-term follow-up. Otolaryngol Head Neck Surg 2007;136:597-604.

17. Kramer SE, Kapteyn TS, Kuik DJ, et al. The association of hearing impairment and chronic diseases with psychosocial health status in older age. J Aging Health 2002;14:122-37.

18. Jung $D$, Bhattacharyya N. Association of hearing loss with decreased employment and income among adults in the United States. Ann Otol Rhinol Laryngol 2012;121:771-5.

19. Lin FR, Metter EJ, O’Brien RJ, et al. Hearing loss and incident dementia. Arch Neurol 2011;68:214-20.

20. Monzani D, Galeazzi GM, Genovese E, et al. Psychological profile and social behaviour of working adults with mild or moderate hearing loss. Acta Otorhinolaryngol Ital 2008;28:61.

21. Li CM, Zhang X, Hoffman HJ, et al. Hearing impairment associated with depression in US adults, National Health and Nutrition Examination Survey 2005-2010. JAMA Otolaryngol Head Neck Surg 2014;140:293-302.

22. Francis HW, Chee $\mathrm{N}$, Yeagle J, et al. Impact of cochlear implants on the functional health status of older adults. Laryngoscope 2002;112:1482-8.

23. Bond M, Mealing S, Anderson R, et al. The effectiveness and costeffectiveness of cochlear implants for severe to profound deafness in children and adults: a systematic review and economic model. Health Technol Assess 2009;13:1-330.

24. Sorkin DL, Buchman CA. Cochlear implant access in six developed countries. Otol Neurotol 2016;37:e161-4.

25. Sorkin DL. Cochlear implantation in the world's largest medical device market: utilization and awareness of cochlear implants in the United States. Cochlear Implants Int 2013;14(Suppl1):S12-S4.

26. National Institute for Health Clinical Excellence. Cochlear Implants for Children and Adults with Severe to Profound Deafness. London, UK: National Institute for Health and Clinical Excellence, 2009.

27. British Cochlear Implant Group. Cl activity 2017; Annual UK update powerpoint. Birmingham, UK: British Cochlear Implant Group, 2018. 
28. Raine C. Cochlear implants in the United Kingdom: awareness and utilization. Cochlear Implants Int 2013;14(Suppl 1):S32-7.

29. Looi V, Bluett C, Boisvert I. Referral rates of postlingually deafened adult hearing aid users for a cochlear implant candidacy assessment. Int J Audiol 2017;56:919-25.

30. Welfare AloHa. ed. AlHW National Hospital Morbidity Database: procedures and healthcare interventions 2013-14 to 2014-15. 8th edn. Australia: Welfare AloHa, 2018.

31. Welfare AloHa. ed. AlHW National Hospital Morbidity Database: procedures and healthcare interventions, 2011-12 to 2012-13. 7th edn. Australia: Welfare AloHa, 2018.

32. Cohen SM, Labadie RF, Haynes DS. Primary care approach to hearing loss: the hidden disability. Ear Nose Throat J 2005;84:26.

33. Raine C, Atkinson H, Strachan DR, et al. Access to cochlear implants: Time to reflect. Cochlear Implants Int 2016;17(Suppl 1):42-6.

34. Coelho DH, Yeh J, Kim JT, et al. Cochlear implantation is associated with minimal anesthetic risk in the elderly. Laryngoscope 2009;119:355-8.

35. Labadie RF, Carrasco VN, Gilmer $\mathrm{CH}$, et al. Cochlear implant performance in senior citizens. Otolaryngol Head Neck Surg 2000;123:419-24.

36. Shin YJ, Fraysse B, Deguine O, et al. Benefits of cochlear implantation in elderly patients. Otolaryngol Head Neck Surg 2000;122:602-6.

37. Davis A, Smith P, Ferguson M, et al. Acceptability, benefit and costs of early screening for hearing disability: a study of potential screening tests and models. Health Technol Assess 2007;11:1-294.

38. Öberg M, Marcusson J, Nägga K, et al. Hearing difficulties, uptake, and outcomes of hearing aids in people 85 years of age. Int $J$ Audiol 2012:51:108-15.

39. Laplante-Lévesque A, Hickson L, Worrall L. Factors influencing rehabilitation decisions of adults with acquired hearing impairment. Int J Audiol 2010;49:497-507.

40. Wallhagen MI. The stigma of hearing loss. Gerontologist 2010;50:66-75

41. Barnett M, Hixon B, Okwiri N, et al. Factors involved in access and utilization of adult hearing healthcare: a systematic review. Laryngoscope 2017;127:1187-94.

42. Cox RM, Alexander GC, Gray GA. Hearing aid patients in private practice and public health (Veterans Affairs) clinics: are they different? Ear Hear 2005;26:513-28.

43. Tong A, Sainsbury P, Craig J. Consolidated criteria for reporting qualitative research (COREQ): a 32-item checklist for interviews and focus groups. Int J Qual Health Care 2007;19:349-57.

44. Cochlear Ltd. Funding options for Cochlear Implants in Australia. 2017 https://cicadaqldcomau/wp-content/uploads/2017/02/ Funding_Options_for_Cochlear_Implants_Australia_Final_LRpdf

45. Foteff C, Kennedy S, Milton AH, et al. Cost-utility analysis of cochlear implantation in Australian adults. Otol Neurotol 2016;37:454-61.

46. British Cochlear Implant Group. How do I get one?UK: BCIG. 2017 http://www.bcig.org.uk/assessed/get-one/

47. Fusch PI, Ness LR. Are we there yet? Data saturation in qualitative research. The Qualitative Report 2015;20:1408.

48. Rapport F, Shih P, Mitchell R, et al. Better evidence for earlier assessment and surgical intervention for refractory epilepsy (The BEST study): a mixed methods study protocol. BMJ Open 2017;7:e017148

49. Lian OS, Rapport F. Life according to ME: caught in the ebb-tide. Health 2016;20:578-98.

50. Rapport F, Jerzembek G, Seagrove A, et al. Evaluating innovations in the delivery and organization of endoscopy services in England and Wales. Qual Health Res 2010;20:922-30.
51. Patton MQ. Qualitative research. New Jersey, USA: John Wiley \& Sons, Ltd, 2005

52. Robinson N. The use of focus group methodology--with selected examples from sexual health research. J Adv Nurs 1999;29:905-13.

53. Kitzinger J. Focus group research: using group dynamics. In: Holloway I, ed. Qualitative research in health care. UK: McGraw-Hill Education, 2005

54. Folch-Lyon E, Trost JF. Conducting focus group sessions. Stud Fam Plann 1981;12:443-9.

55. Bloor M. Focus groups in social research. UK: Sage, 2001.

56. Hammersley M, Atkinson P. Ethnography: principles in practice. 3rd edn. UK: Routledge, 2007

57. Krueger RA, Casey MA. Designing and conducting focus group interviews. Social analysis, selected tools and techniques. Washington, DC, USA: The World Bank, 2002:4-24.

58. Balch Gl, Mertens DM. Focus group design and group dynamics: lessons from deaf and hard of hearing participants. Am J Eval 1999;20:265-77.

59. Napier J, Barker R. Accessing university education: perceptions, preferences, and expectations for interpreting by deaf students. $J$ Deaf Stud Deaf Educ 2004;9:228-38.

60. Braun V, Clarke V. Using thematic analysis in psychology. Qual Res Psychol 2006;3:77-101.

61. Iredale R, Rapport F, Sivell S, et al. Exploring the requirements for a decision aid on familial breast cancer in the UK context: a qualitative study with patients referred to a cancer genetics service. J Eval Clin Pract 2008;14:110-5.

62. Rapport F, Iredale R, Jones W, et al. Decision aids for familial breast cancer: exploring women's views using focus groups. Health Expect 2006;9:232-44.

63. Rapport F, Shih P, Bierbaum M, et al. Schema analysis of qualitative data: a team based approach. In: Liamputtong P, ed. Handbook of research methods in health social sciences. Singapore: Springer, 2018.

64. Taylor JK, Cihon C. Statistical techniques for data analysis. Florida, USA: CRC Press, 2004.

65. Denzin NK, Lincoln YS. Handbook of qualitative research. California, USA: Sage, 2005.

66. Liamputtong P. Qualitative research methods. Australia: OUP Australia \& New Zealand, 2013.

67. Pope C, Ziebland S, Mays N. Qualitative research in health care. Analysing qualitative data. BMJ 2000;320:114-6.

68. Robson C. Real world research: a resource for users of social research methods in applied settings. 3rd edn. West Sussex: John Wiley \& Sons, 2011.

69. Flick U. Triangulation in qualitative research. In: Flick U, von Kardoff $\mathrm{E}$, Steinke I, eds. A companion to qualitative research. California, USA: Sage, 2004:178-83.

70. Golafshani N. Understanding reliability and validity in qualitative research. The Qualitative Report 2003;8:597-606.

71. Jick TD. Mixing qualitative and quantitative methods: triangulation in action. Adm Sci Q 1979;24:602-11.

72. Lincoln YS, Guba EG. But is it rigorous? Trustworthiness and authenticity in naturalistic evaluation. New Directions for Program Evaluation 1986:1986:73-84.

73. Berends L, Johnston J. Using multiple coders to enhance qualitative analysis: the case of interviews with consumers of drug treatment. Addict Res Theory 2005;13:373-81.

74. National Health Medical Research Council. National statement on ethical conduct in human research: National Health and Medical Research Council. Australia: National Health Medical Research Council, 2007. 